\title{
Editorials
}

\section{Is it getting easier to obtain antibiotics in the UK?}

Public knowledge and understanding of antibiotics are poor, with only $56 \%$ of the UK general public aware that antibiotics cannot kill viruses, ${ }^{1}$ and those with poorer knowledge are more likely to use them.' Clinical assessment therefore remains a vital step in assessing the need for and safety of antibiotics, and an essential opportunity for education about appropriate use, antimicrobial resistance (AMR), and self-care.

In the UK, antibiotics are, with very few exceptions, only prescribable by doctors or other health professionals with prescribing qualifications. This has meant that, until recently, access to antibiotics has been possible only through face-to-face medical assessment in primary or secondary care, providing a significant disincentive to seeking antibiotics unnecessarily.

Inappropriate prescribing of antibiotics in UK primary care remains of concern, ${ }^{2}$ but antimicrobial stewardship (AMS) initiatives are having a measurable effect, with prescribing rates falling in response to interventions. ${ }^{3}$ However, novel routes to obtaining antibiotics, associated with either a lower threshold for prescribing or issuing of antibiotics without medical assessment, undermine these strategies and are likely to increase inappropriate use.

\section{A LOWER THRESHOLD FOR PRESCRIBING ANTIBIOTICS: \\ TELEPHONE AND ONLINE CONSULTING}

Workforce shortage and increasing demand in primary care has led many NHS providers to try alternatives to face-to-face appointments as a means of managing workload. Telephone consultations are now commonplace in NHS primary care, and continued government pressure for greater integration of technology means that many practices give patients the option of sending queries to their GP via email or online forms. Public demand for more convenient access to health care has also led to a proliferation of private providers, both pharmacies and GPs, offering, for a fee, remote clinical advice and treatments via online forms, computer algorithms, and video consultations. These providers also offer their services to NHS patients in some areas.

Although these alternative forms of consulting offer greater convenience to patients, a number of factors may make

\section{novel routes to obtaining antibiotics ... undermine}

[antimicrobial stewardship] strategies and are likely to increase inappropriate use.

prescription of antibiotics more likely than in traditional face-to-face settings. ${ }^{4}$

The inability to examine patients when conducting consultations remotely will have an important impact on clinical judgement and assessment of risk. Doctors are significantly less satisfied with their examination via video compared with faceto-face consultations, ${ }^{5}$ and testing with video vignettes has shown that doctors find upper respiratory tract infections one of the most difficult conditions to assess via video consultation. ${ }^{6}$ Reduced availability of diagnostic testing such as laboratory urine and throat swab culture when consulting remotely may further contribute to diagnostic uncertainty, leading clinicians to prescribe just in case. ${ }^{7}$ Clinicians engaging in telephone or online consulting are also likely to feel pressure to 'close' the consultation rather than bring the patient in to the surgery, making prescribing of antibiotics more likely.

In private sector online consulting GPs additionally lack the usual safety nets available in NHS primary care, with limited background information on patients and reduced scope for followup. Consequently, they may well be more likely to prescribe antibiotics. Furthermore, perceived patient expectations, likely to be high in private consultations, ${ }^{9}$ are known to increase prescribing: ${ }^{10}$ patients who obtain antibiotics also give more positive feedback an important factor for all providers in a competitive marketplace. ${ }^{1}$

Evidence regarding levels of prescribing of antibiotics via telephone and online consulting is limited. However, it has been reported that antibiotics are frequently prescribed in these settings. ${ }^{12,13}$ Several studies have demonstrated significantly increased likelihood of antibiotic prescribing where patients consult health professionals via telephone or video compared with faceto-face settings for a variety of commonly seen infections including pharyngitis, bronchitis, sinusitis, and urinary tract infections $7,14,15$

\section{ANTIBIOTICS WITHOUT PRESCRIPTION SALE OF ANTIBIOTICS ONLINE}

Another threat to AMS is the sale of antibiotics without a prescription by online retailers based outside the UK. ${ }^{16}$ Although the extent of this activity is difficult to quantify, a study in 2009 identified 136 unique online retailers prepared to ship antibiotics to consumers in the US without prescription; ${ }^{17}$ it is very likely that availability has grown substantially since then. ${ }^{18}$ Not only does this increase the likelihood of unnecessary antibiotics use, but also exposes patients to potentially significant risks. Allowing patients to bypass medical assessment completely, such retailers often offer consumers a wide choice of antibiotics, with limited or no checks of suitability and safety prior to sale. ${ }^{16}$ Lack of medical assessment may delay recognition of important symptoms or signs, while the antibiotics may be inappropriate in choice, dose, or duration. ${ }^{16}$ Furthermore, the existence of counterfeit antibiotics is a problem worldwide, and antibiotics obtained from online retailers may be of unclear provenance. ${ }^{18}$

EU data suggest that the internet is not currently an important source of antibiotics without a prescription. ${ }^{19}$ However, online availability is increasing, and its impact may differ in the UK; in other EU states, significant over-the-counter sales of antibiotics take place in pharmacies, ${ }^{19}$ whereas in the UK availability of antibiotics from physical pharmacy stores without a prescription is almost non-existent. Perceived availability of antibiotics over-thecounter without a prescription is known to increase the probability of self-medication, ${ }^{20}$ often for conditions not requiring antibiotics such as viral respiratory tract infections. ${ }^{19}$ As public awareness of availability online without prescription increases, it seems very likely that self-medication with antibiotics obtained online, and their use for inappropriate indications, will also increase. 


\section{IMPLICATIONS FOR UK POLICY AND PRACTICE}

There is clearly demand for the rapid and convenient access to healthcare advice offered by new alternatives to face-to-face medical consultations. However, there is a substantial risk that, whether via NHS or private providers, these may result in easier availability of antibiotics and increased inappropriate use, through a lower threshold for prescribing. Research is needed to quantify the extent of prescribing by these routes. However, with the potential of telephone and online consulting to reduce workload pressure in NHS primary care debatable, further implementation should be avoided until evidence of possible unintended consequences for AMR is clearer.

There is also a pressing need for action in relation to online pharmacies selling antibiotics without medical assessment, with development of effective means of monitoring this activity essential. The impact of availability of antibiotics over-the-counter without prescription in other EU countries suggests that the sale of antibiotics online is likely to increase inappropriate use, while exposing patients to the risk of taking poor-quality or counterfeit medications. In 2015, the Review on Antimicrobial Resistance highlighted the problem of sale of antimicrobial drugs on the internet without prescription, calling for globally coordinated regulatory activity to address this. ${ }^{18}$ Regulators and professional bodies must work together with government to find solutions, including legislation, while engaging with the public to highlight the risks of self-medication.

\section{ADVERTISING REGULATIONS}

Finally, various pharmacies and private online GP services appear to use sponsored links to place themselves at the top of internet searches relating to antibiotics. Sending a message of easy accessibility of antibiotics, this further undermines AMS strategies. Formal regulation of advertising of healthcare services that may be interpreted as promoting inappropriate use of antibiotics, or where access to antibiotics appears to be used as a marketing tool, is essential.

\section{Benedict Hayhoe,}

NIHR Clinical Lecturer in Primary Care, Department of Primary Care and Public Health, Imperial College London, London.

\section{Geva Greenfield,}

Research Fellow in Public Health, Department of Primary Care and Public Health, Imperial College London, London.

\section{Azeem Majeed,}

Professor of Primary Care, Department of Primary Care and Public Health, Imperial College London, London.

\section{Funding}

This research was supported by the Imperial NIHR Biomedical Research Centre and the National Institute for Health Research Collaboration for Leadership in Applied Health Research and Care Northwest London (NIHR CLAHRC NWL). The views expressed in this article are those of the author(s) and not necessarily those of the NHS, the NIHR, or the Department of Health and Social Care.

\section{REFERENCES}

1. European Commission. TNS Opinion and Social. Special Eurobarometer 445. Report. Antimicrobial resistance. Brussels: European Commission, 2016. https://ec.europa.eu/health/ amr/sites/amr/files/eb445_amr_generalreport en.pdf (accessed 5 Jan 2019).

2. Smieszek T, Pouwels KB, Dolk FCK, et al. Potential for reducing inappropriate antibiotic prescribing in English primary care. $J$ Antimicrob Chemother 2018; 73(Suppl_2): ii36-43.

3. Bou-Antoun S, Costelloe C, Honeyford K, et al. Age-related decline in antibiotic prescribing for uncomplicated respiratory tract infections in primary care in England following the introduction of a national financial incentive (the Quality Premium) for health commissioners to reduce use of antibiotics in the community: an interrupted time series analysis. J Antimicrob Chemother 2018; 73(10): 2883-2892.

4. Peters L, Greenfield G, Majeed A, Hayhoe B. The impact of private online video consulting in primary care. J R Soc Med 2018; 111(5): 162-166.

5. Stahl JE, Dixon RF. Acceptability and willingness to pay for primary care videoconferencing: a randomized controlled trial. J Telemed Telecare 2010; 16(3): 147-151.

6. Jiwa M, Meng X. Video consultation use by Australian general practitioners: video vignette study. J Med Internet Res 2013; 15(6): e117.

7. Gordon AS, Adamson WC, DeVries AR. Virtual visits for acute, nonurgent care: a claims analysis of episode-level utilization. J Med Internet Res 2017; 19(2): e35.

8. Bjørnsdottir I, Hansen EH. Telephone prescribing of antibiotics. General practitioners views and reflections. Eur J Public Health 2001 11(3): 260-263.

9. Ab Rahman N, Teng CL, Sivasampu S Antibiotic prescribing in public and private practice: a cross-sectional study in primary care clinics in Malaysia. BMC Infect Dis 2016; 16: 208

10. Teixeira Rodrigues A, Roque F, Falcão $A$, et al. Understanding physician antibiotic prescribing behaviour: a systematic review of qualitative studies. Int J Antimicrob Agents 2013; 41(3) 203-212.

\section{ADDRESS FOR CORRESPONDENCE}

\section{Benedict Hayhoe}

Department of Primary Care and Public Health, Imperial College London, Charing Cross Campus, The Reynolds Building, St Dunstan's Road, London W6 8RP, UK.

\section{Email: b.hayhoedimperial.ac.uk}

\section{Provenance}

Freely submitted; externally peer reviewed.

DOI: https://doi.org/10.3399/bjgp19X700829
11. Ashworth $M$, White $P$, Jongsma $H$, et al. Antibiotic prescribing and patient satisfaction in primary care in England: cross-sectional analysis of national patient survey data and prescribing data. Br J Gen Pract 2016; DOI: https://doi.org/10.3399/bjgp15X688105.

12. Ewen E, Willey VJ, Kolm P, et al. Antibiotic prescribing by telephone in primary care. Pharmacoepidemiol Drug Saf 2015; 24(2): 113-120.

13. Brunett PH, DiPiero A, Flores C, et al. Use of a voice and video internet technology as an alternative to in-person urgent care clinic visits. J Telemed Telecare 2015; 21(4): 219-226.

14. Mehrotra A, Paone S, Martich GD, et al. A comparison of care at e-visits and physician office visits for sinusitis and urinary tract infection. JAMA Intern Med 2013; 173(1): 72-74.

15. Uscher-Pines L, Mulcahy A, Cowling D, et al. Access and quality of care in direct-toconsumer telemedicine. Telemed J E Health 2016; 22(4): 282-287.

16. Boyd SE, Moore LSP, Gilchrist M, et al. Obtaining antibiotics online from within the UK: a cross-sectional study. J Antimicrob Chemother 2017; 72(5): 1521-1528.

17. Mainous AG 3rd, Everett CJ, Post RE, et al. Availability of antibiotics for purchase without a prescription on the internet. Ann Fam Med 2009; 7(5): 431-435.

18. Review on Antimicrobial Resistance. Safe, secure and controlled: managing the supply chain of antimicrobials. London: Wellcome Trust and HM Government, 2015. https://amr-review.org/sites/default/files/ SafeSecureandControlledShortPaper.pdf (accessed 20 Dec 2018).

19. Paget J, Lescure D, Versporten A, et al. Antimicrobial resistance and causes of nonprudent use of antibiotics in human medicine in the EU. Brussels: European Commission, 2017. https://ec.europa.eu/health/amr/sites/ amr/files/amr arna report_20170717_en.pdf laccessed 20 Dec 2018).

20. Grigoryan L, Burgerhof JG, Degener JE, et al. Determinants of self-medication with antibiotics in Europe: the impact of beliefs, country wealth and the healthcare system. $J$ Antimicrob Chemother 2008; 61(5): 1172-1179. 